\title{
Effect of Cocrystal Behavior on Sensitivity and Thermal Decomposition Mechanisms of CL-20/HMX via Molecular Dynamics Simulations
}

\author{
Yizheng Fu, ${ }^{1,2^{*}}$ Penghui Zhao, ${ }^{1,2}$ Luxia Yang, ${ }^{3}$ Ruizheng Miao, ${ }^{4}$ Congyun Zhang, ${ }^{1,2}$ Zhanhu Guo, ${ }^{5}$ and Yaqing Liu ${ }^{1,2^{*}}$
}

Molecular simulations were performed to investigate the effect of cocrystal behavior on the properties of 2,4,6,8,10,12-hexanitro2,4,6,8,10,12-hexaazaisowurtzitane/1,3,5,7-tetranitro-1,3,5,7-tetrazacyclooctane (CL-20/HMX) explosive. The sensitivity, binding energy and the mechanical properties of the cocrystals were simulated by molecular dynamics (MD) with COMPASS force field, and the thermal decomposition mechanisms were studied by reactive molecular dynamics (RMD) simulations with ReaxFF/lg. The results showed the binding energy between CL-20 and HMX in cocrystal structure was larger than mixture structure, indicating the more excellent stability of the former. Compared with pure crystal of CL-20, the value of tensile $(E)$, bulk $(K)$, shear $(G)$ moduli of CL-20/HMX cocrystals and the physical mixture all decreased. Thus, both mixture and cocrystal structure reduced the stiffness and increased flexibility of CL-20, and they showed better security. RMD simulation showed that the potential energy change of mixture was larger than that of the cocrystal at the earlier thermal decomposition stage. However, the potential energy drops faster in cocrystal than in mixture in the later process due to the interactions of the products from CL-20 decomposition. The main products were $\mathrm{NO}_{2}, \mathrm{~N}_{2}, \mathrm{NO}, \mathrm{H}_{2} \mathrm{O}, \mathrm{HONO}, \mathrm{HON}$ and $\mathrm{CO}_{2}$.

Keywords: CL-20/HMX; Sensitivity; Mechanical properties; Thermal decomposition mechanism

Received 6 August 2018, Accepted 12 October 2018

DOI: $10.30919 / \mathrm{esmm} 5 \mathrm{f} 126$

\section{Introduction}

2,4,6,8,10,12-hexanitro-2,4,6,8,10,12-hexaazaisowurtzitane, commonly referred to CL-20, with its rigid isowurtzitane cage and nitro groups being attached to the bridging nitrogen atoms, is the most powerful explosive material presently available, and has a promising prospect of application. ${ }^{1-3}$ However, the highly energetic materials suffer from the relatively poor safety and high economic cost, which seriously hinder development and application of CL-20, ${ }^{4,5}$

The cocrystallization of explosive, which is a multicomponent crystal of several neutral explosive molecules formed by noncovalent interactions including $\mathrm{H}$-bond, electrostatic interaction in a defined ratio, ${ }^{6,7}$ has drawn great attention because it can satisfy the demands for both power and safety somewhat. Recently a lot of cocrystal explosives have been synthesized and characterized, such as CL-20/1,3-dinitrobenzene $(\mathrm{DNB})^{8}$, CL-20/2,4,6-trinitrotoluene (TNT), ${ }^{9}$ CL-20/Benzotrifuroxan (BTF), ${ }^{10}$ CL-20/1,3,5-triamino-

${ }^{I}$ School of Materials Science and Engineering, North University of China, Taiyuan 030051, China

${ }^{2}$ Shanxi Province Key Laboratory of Functional Nanocomposites, North University of China, Taiyuan 030051, China

${ }^{3}$ Business College of Shanxi University, Taiyuan 030051, China

${ }^{4} X i$ ' an North Huian Chemical Industries Co. Ltd. , Xi' an 710302, China

${ }^{5}$ Integrated Composites Laboratory (ICL), Department of Chemical \& Biomolecular Engineering, University of Tennessee, Knoxville, Tennessee 37996, USA

*E-mail: fuyizheng@nuc.edu.cn; lyq@nuc.edu.cn 2,4,6-trinitrobenzene (TATB) ${ }^{11}$ and CL-20/caprolactam (CPL). ${ }^{12}$ Bolton et $\mathrm{al}^{13}$ cocrystallized CL-20 with the cheap and insensitive explosive HMX (1,3,5,7-tetranitro-1,3,5,7-tetrazacyclooctane) to form a novel cocrystal explosive. The prepared cocrystal explosive with a lower sensitivity, makes up the deficiency of conventional energetic materials.

Energetic materials may release a lot of energy in a short time through complex chemical reactions under extreme conditions, which would bring out some challenge in time and space. Thus, it is difficult to provide some microscopic information from the atomic or molecular scale in current experimental conditions. Molecular dynamics (MD) simulation is a newly analytical tool used to describe the detailed information at atomic level and femtosecond scale. Especially, the reaction molecular dynamics (RMD) simulation method based on the reactive force field, as the bridge to connect quantum chemistry and molecular dynamics methods, could provide a method to investigate property and chemical reaction of energetic materials in atomic level. They can not only facilitate the understanding of reaction mechanism of energetic materials in extreme conditions, but also help to obtain the energy release law. ${ }^{13}$

Rom $^{14}$ studied the decomposition mechanism of hot liquid nitromethane at various compressions via ReaxFF molecular dynamics simulations. It has been demonstrated that there were differences of thermal decomposition between higher and lower compression. Han ${ }^{15}$ showed that the reaction path was seriously influenced by the temperature through investigating thermal decomposition and subsequent reaction of the energetic material nitromethane $\left(\mathrm{CH}_{3} \mathrm{NO}_{2}\right)$ using molecular dynamics with ReaxFF forcefield. Strachan16 reported the thermal induced chemistry in 
cyclotrimethylene trinitramine (RDX) at different temperatures and densities. With time evolution, the change of potential energy can be described well through a single exponential function that increases with declining density and shows an Arrhenius temperature dependence. Weismiller ${ }^{17}$ studied the chemical kinetics of ammonia borane oxidation. The results elucidated the pertinent chemical pathways, intermediate species and chemical kinetic mechanism. Strachan ${ }^{18}$ demonstrated shock propagation of RDX through nonequilibrium MD simulations at different collision velocities, high impact velocities $(>6 \mathrm{~km} / \mathrm{s}$ ). The RDX molecules decomposed and reacted to form all kinds of small molecules in very short time scales $(<3 \mathrm{ps})$, and at lower velocities, only $\mathrm{NO}_{2}$ was formed in agreement with experiment results. Zhang ${ }^{19}$ studied the initial thermal decomposition pathways of supercell structure and signal molecule of CL-20 explosive at various densities and temperatures by RMD. The results showed that the density and temperature affected the rate of pyrolysis, but exerted no effects on pyrolysis mechanism. Compared with the pure energetic materials, fewer references were reported in CL-20 eutectic of energetic materials by RMD simulation. $\mathrm{Liu}^{20}$ reported the initial thermal decomposition of condensed phase CL20/TNT cocrystal under high temperature. Products identification analysis showed that the main products were $\mathrm{NO}_{2}, \mathrm{NO}, \mathrm{CO}_{2}, \mathrm{~N}_{2}, \mathrm{H}_{2} \mathrm{O}, \mathrm{HON}, \mathrm{HNO}_{3}$. Goddard ${ }^{21}$ separately compared the thermal decomposition process of the TNT/CL-20 cocrystal with that of pure crystals of TNT, CL-20, a simple physical mixture of TNT and CL-20. They found that cocrystal exhibited a lower decomposition rate than pure CL-20, suggesting the decrease in sensitivity of the cocrystal. These all could provide us with a rational method to study the thermal decomposition mechanism of CL-20/HMX cocrystal.

In this study, molecular dynamics simulation method was employed to study the pure CL-20, HMX, CL-20/HMX mixture and
CL-20/HMX cocrystal with COMPASS (condensed-phase optimized molecular potentials for atomistic simulation studies) forcefield ${ }^{22}$, and the sensitivity and mechanical property of these structures were analyzed. Then, the thermal decomposition of these systems were studied by RMD with ReaxFF/lg forcefield. ${ }^{23}$ Therefore, the effect cocrystal behavior on the thermal decomposition mechanism of energetic materials was obtained.

\section{Modelling and simulation details}

\subsection{Modelling construction}

CL-20 and HMX molecular structures are displayed in Fig. 1. In this paper, Materials Studio 6.0 software was used to build all molecular structures, the initial unit cell structures of cocrystal CL-20/HMX, ${ }^{13}$ CL-20 $0^{24}$ and $\mathrm{HMX}^{25}$ were taken from Cambridge Crystallographic Data Cenre. The CL-20/HMX cocrystal with supercell $2 \times 3 \times 2$ (Fig. 2a), was constructed for avoiding the influence of temperature and heating rate fluctuation on reaction and obtaining more accurate statistical results. For comparison, pure CL-20 with supercell $3 \times 2 \times 2$ (Fig. 2b), pure HMX with supercell $2 \times 3 \times 2$ (Fig. 2c) and hybrid CL20/HMX mixture (Fig. 2d) with molar ratio 2:1 were constructed. The mixture was constructed by using Build Layers tool and put $3 \times 4 \times 2$ CL-20 supercell and $4 \times 3 \times 1$ HMX supercell into a supercell system. a<smiles>O=NN(C1C2C3N([N+](=O)[O-])C(C1N([N+](=O)[O-])N3[N+](=O)[O-])N2[N+](=O)[O-])[N+](=O)[O-]</smiles>

b<smiles>O=[N+]([O-])N1CN([N+](=O)[O-])C[N+]([N+](=O)[O-])([N+](=O)[O-])C1</smiles>

Fig. 1 The molecular formulas for CL-20 (a) and HMX (b).
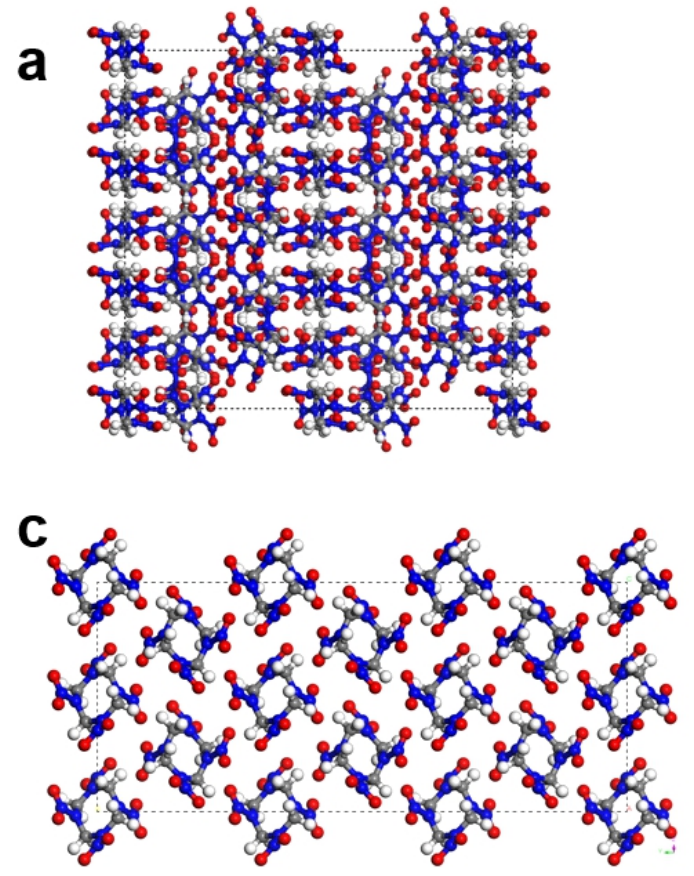
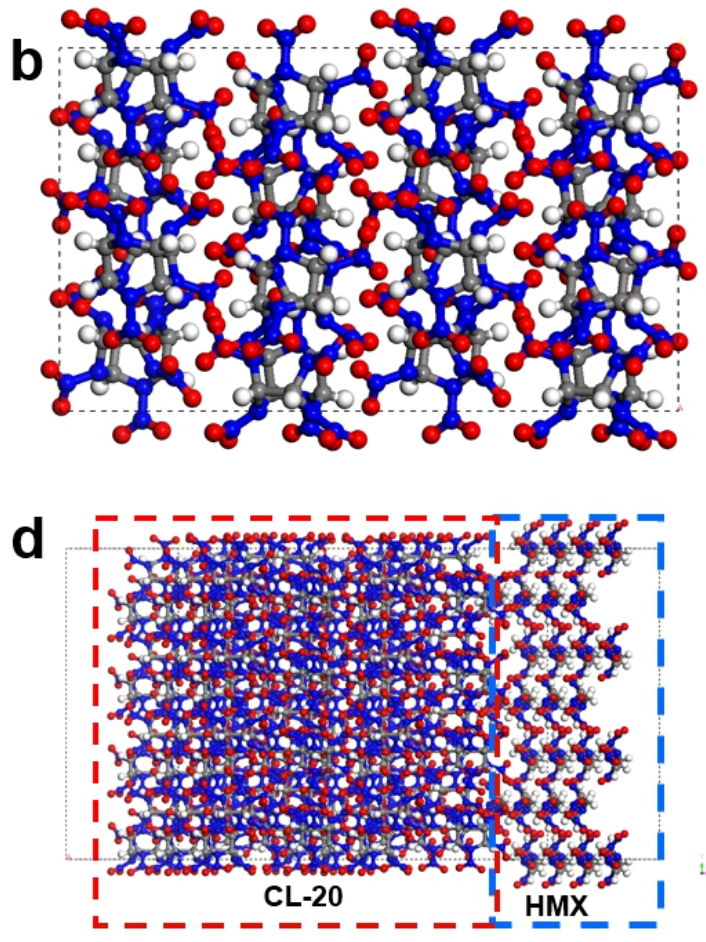

Fig. 2 The molecular structures for CL-20/HMX cocrystal(a), Pure CL-20(b), Pure HMX(c) and CL-20-HMX mixture(d). 
Table 1 The $L_{\max }$ and $L_{\text {ave }}$ of trigger bonds of N-NO ${ }_{2}$ in CL-20 and N-NO $\mathrm{NO}_{2}$ in $\mathrm{HMX}(\AA)$.

\begin{tabular}{|c|c|c|c|c|}
\hline System & $\begin{array}{c}L_{\text {ave }} \text { of } \mathrm{N}-\mathrm{NO}_{2} \\
\quad \text { of CL-20 }\end{array}$ & $\begin{array}{c}L_{\text {ave }} \text { of } \mathrm{N}^{-\mathrm{NO}_{2}} \text { of } \\
\mathrm{HMX}\end{array}$ & $\begin{array}{c}L_{\max } \text { of } \mathrm{N}-\mathrm{NO}_{2} \text { of } \\
\text { CL-20 }\end{array}$ & $\begin{array}{c}L_{\max } \text { of } \mathrm{N}-\mathrm{NO}_{2} \\
\text { of HMX }\end{array}$ \\
\hline Pure CL-20 & 1.395 & $-\cdots$ & 1.551 & $\begin{array}{l}--- \\
---\end{array}$ \\
\hline Pure HMX & ---- & 1.397 & ---- & 1.518 \\
\hline CL-20/HMX Mixture & 1.395 & 1.399 & 1.548 & 1.534 \\
\hline CL-20/HMX Cocrystal & 1.392 & 1.398 & 1.537 & 1.541 \\
\hline
\end{tabular}

\section{2 Simulation details}

Each unit cell was subjected to the energy minimization through smart minimizing method to remove the local non-equilibrium structure. To further relax local hot-spots and achieve better equilibrium of the system, MD simulations were performed at $300 \mathrm{~K}$ for $500 \mathrm{ps}$ in the NVT canonical ensemble, and at $300 \mathrm{~K}, 1$ bar for $500 \mathrm{ps}$ in the NPT ensemble, successively. Trajectories were saved every 1 ps and the final 50 ps configurations were used to analyze the bond length, binding energy and mechanical property. Temperature and pressure were controlled by the Andersen ${ }^{26}$ and Berendsen's ${ }^{27}$ methods respectively. The Ewald summation ${ }^{28}$ was used to calculate the Coulombic interactions. The atom-based summation ${ }^{29}$ was applied to calculate van der Waals's interactions with a cutoff distance of 0.95 $\mathrm{nm}$, a spline width of $0.1 \mathrm{~nm}$ and a buffer width of $0.5 \mathrm{~nm}$. The time step was set as $1 \mathrm{fs}$. During the whole simulation process, COMPASS forcefield was used for computing interatomic interactions.

large-scale atomic/molecular massive parallel simulator (Lammps) software was chosen to investigate the thermal decomposition of cocrystal, pure crystals, and simple mixtures. RMD simulations with ReaxFF/lg forcefield were performed at $300 \mathrm{~K}$ for $500 \mathrm{ps}$ in NVT ensemble, and at $300 \mathrm{~K}, 1$ bar for $10 \mathrm{ps}$ in the NPT ensemble, successively. Finally, at $2000 \mathrm{~K}$, RMD simulation was performed for $50 \mathrm{ps}$ in NVT ensemble, and Berendsen method was used for the temperature and pressure controlling. Therein, periodic boundary condition was chosen, and coupling parameter and the whole simulation time were set as $100 \mathrm{fs}$, respectively. Trajectories were saved every $50 \mathrm{fs}$ and band orders were set as 0.3 .

\section{Results and discussion}

\subsection{The trigger bonds affected by cocrystalization and mixture}

A comparison of the bond orders of trigger bonds, a criterion ${ }^{30}$ can be used to judge the relative stability and security of energetic compounds. As the "principle of the smallest bond order" (PSBO) indicates, for the trigger bond of energetic compound, its bond order is closely related to the sensitivity of the compound. Namely, the smaller the order is, the bigger the sensitivity is. This is especially suitable for the compound with similar molecular structures. Generally, the decrease of the bond order will lead to the increase of the bond length, and vice versa. Since the bond order and length decide the strength of the energetic compound, the statistical distribution of bond length obtained through classical MD simulation can be employed to evaluate the compound stability. The longer the bond length is, the more likely the bond is to break. This will enhance the molecule's activity and decomposition. The molecule with the maximum bond length has the greatest activity and can be most easily activated and decomposed.

The bond length of $\mathrm{N}-\mathrm{NO}_{2}$ trigger bond in CL-20 and $\mathrm{N}-\mathrm{NO}_{2}$ trigger bond in HMX molecules were calculated. Table 1 shows the average bond length $\left(L_{\text {ave }}\right)$ and maximum bond length $\left(L_{\max }\right)$ of trigger bonds in different simulation systems. It is observed that $\mathrm{CL}$ 20/HMX with mixture and cocrystal modes exert a slight influence on the average bond length comparing to pure CL-20 or pure HMX, whereas have an obvious effect on the maximum bond length. The mixture and CL-20/HMX cocrystal made the maximum bond length of trigger bond $\mathrm{N}-\mathrm{NO}_{2}$ in CL-20 become shorter and $\mathrm{N}-\mathrm{NO}_{2}$ in $\mathrm{HMX}$ elongated. The sensitivity is closely connected with the maximum bond length of trigger bond, so the mixture and CL-20/HMX cocrystal reduced the sensitivity of CL-20 and increased the sensitivity of HMX. Otherwise, effects of cocrystal on the trigger bonds are more obvious than that of mixture.

\subsection{The binding energy}

The format in this paragraph may be different with others. the negative value of the intermolecular interaction energy $\left(E_{\text {inter }}\right), E_{\text {bind }}=$ $E_{\text {inter }}$, which can well reveal the interaction between the two components $^{31}$. The intermolecular interaction energy was calculated based on the total energies of the whole system and the individual component energy in the system. $E_{\text {bind }}$ between CL-20 and HMX was expressed as Eq. 1 .

$$
E_{\text {bind }}=-E_{\text {inter }}=-\left(E_{\text {tood }}-E_{C L-20}-E_{\mathrm{HMX}}\right)
$$

Where $E_{\text {total }}$ represents the total energy of the whole system, and $E_{\mathrm{CL}-20}$ and $E_{\mathrm{HMX}}$ are the energies of CL-20 and HMX, respectively. Higher binding energy reveals stronger interaction between components. ${ }^{32,33}$

Table 2 shows the binding energies $\left(E_{\text {bind }}\right)$, the van der walls energies $\left(E_{\text {vdW }}\right)$, and the electrostatic energies $\left(E_{\text {elec }}\right)$ of CL-20/HMX cocrystal and their mixtures. The binding between CL-20 and HMX of CL-20/HMX cocrystal is far stronger than CL-20/HMX mixture, which indicates that the CL-20/HMX cocrystal structure is more stable than that of their mixture. As shown in Table 2, with the COMPASS force field, binding energy $E_{\text {bind }}$ is the summation of $E_{\mathrm{vdw}}$ and $E_{\text {elec }}$, and electrostatic portion is the major contributions to the binding energy. Therefore, the cocrystallization changed the interior constitution and structure of CL-20 crystal and resulted in the 
formation of strong intramolecular interaction of the two components, ${ }^{34}$ which all increase the stability of cocrystal explosives, enhance the vibration resistance to external forces, and improve the security of explosives.

Table $2 E_{\text {bind }}, E_{\text {elec }}$ and $E_{\text {vdw }}$ of different systems (kcal $\left./ \mathrm{mol}\right)$.

\begin{tabular}{cccc}
\hline System & $E_{\text {bind }}$ & $E_{\text {elec }}$ & $E_{\text {vdw }}$ \\
\hline CL-20/HMX Cocrystal & 861.57 & 810.28 & 13.08 \\
CL-20/HMX Mixture & 257.18 & 176.74 & 19.67 \\
\hline
\end{tabular}

\subsection{The mechanical property}

Material stress $(\sigma)$ and strain tensors $(\varepsilon)$ are applied to explosive materials to exhibit small deformations when subjected to external forces. Constant stress molecular dynamics simulations were then used to analyze the elastic moduli obtained from the stress-strain behavior of materials when subjected to an applied load. The dependence of the stress on the strain for elastic materials is shown in Eq. 2.

$$
\sigma_{i j}=C_{i j k l} \varepsilon_{k l}
$$

$C_{\mathrm{ijkl}}(\mathrm{i}, \mathrm{j}, \mathrm{k}, \mathrm{l}=1,2,3)$ are the elements of the elastic symmetric matrix. To fully describe the stress-strain behavior of an arbitrary material, a maximum of up to 21 constants are required. The $\sigma$ and $\varepsilon$ are expressed by Eq. 3 and 4 , where $i, m_{\mathrm{i}}, v_{\mathrm{i}}$ and $f_{\mathrm{i}}$ are the number, mass, velocity of the particle and force acting on the particle, respectively. The parameter $V_{0}$ represents the volume of the system.

$$
\begin{array}{r}
\sigma=-\frac{1}{V_{0}}\left[\left(\sum_{i=1}^{N} m_{i}\left(v_{i} v_{i}^{T}\right)\right)+\left(\sum_{i<j} r_{i j} f_{i j}^{T}\right)\right] \\
\varepsilon=\frac{1}{2}\left[\left(h_{0}^{T}\right)^{-1} G h_{0}^{-1}-1\right]
\end{array}
$$

In Eq.4, $h_{0}$ and $h$ represent the matrix of the initial dimension of the structure and the dimension of the deformed structure, respectively. $G$ is the metric tensor $h^{\mathrm{T}} h$.

The Reuss average is used to obtain the effective isotropic compliances of given materials averaged over all orientations. The effective bulk moduli $(K)$ and shear moduli $(G)$ are shown in Eq. 5 and 6 , respectively, where the subscript $\mathrm{R}$ represents the Reuss average. The compliance matrix $S$ stands for the inverse matrix of the elastic symmetric matrix $C$, i.e., $S=C^{1}$. The isotropic linear tensile moduli $(E)$ and Poisson's ratio $(v)$ can be obtained from Eq. 7.

$$
\begin{gathered}
K_{R}=\left[S_{11}+S_{22}+S_{33}+2\left(S_{12}+S_{23}+S_{31}\right)\right]^{-1} \\
G_{R}=5\left[4\left(S_{11}+S_{22}+S_{33}\right)-4\left(S_{12}+S_{23}+S_{31}\right)+\left(S_{44}+S_{55}+S_{66}\right)\right]^{-1} \\
E=2 G(1+v)=3 K(1-2 v)
\end{gathered}
$$

Tensile, bulk and shear moduli reveal the mechanical properties of pure CL-20, HMX, CL-20/HMX cocrystal and CL-20/HMX mixture, which were summarized in Table 3. $E, K$ and $G$ of CL20/HMX cocrystal and mixture were lower than those of CL-20, which indicate that cocrystal and mixture decreased the stiffness and made CL-20 system more flexible and "softer". When the system were subjected to an external force, it can effectively buffer, disperse

\begin{tabular}{|c|c|c|c|c|}
\hline Parameter & CL-20 & HMX & Cocrystal & Mixture \\
\hline Tensile modulus (E) & 9.4 & 7.5 & 2.6 & 6.6 \\
\hline Poisson ratio ( $\mathrm{v}$ ) & 0.3 & 0.3 & 0.4 & 0.3 \\
\hline Bulk modulus $(\underline{\mathrm{K}})$ & 7.3 & 5.3 & 3.9 & 5.4 \\
\hline Shear modulus $(\underline{G})$ & 3.4 & 3.8 & 2.3 & 2.4 \\
\hline$\underline{K} / \mathrm{G}$ & 2.1 & 1.4 & 1.7 & 2.3 \\
\hline
\end{tabular}
force and reduce the friction between the explosive particles, leading to the internal stress distribution more evenly. Thus, the cocrystal and mixture reduce the "hot spot" formation and sensitivity of CL-20 explosive, increasing the security of CL- $20 .^{35}$

Table 3 The mechanical properties of different systems

\subsection{Thermal decomposition}

To study the effect of cocrystal on the thermal decomposition mechanism of CL-20, we employ ReaxFF/lg in RMD simulations to
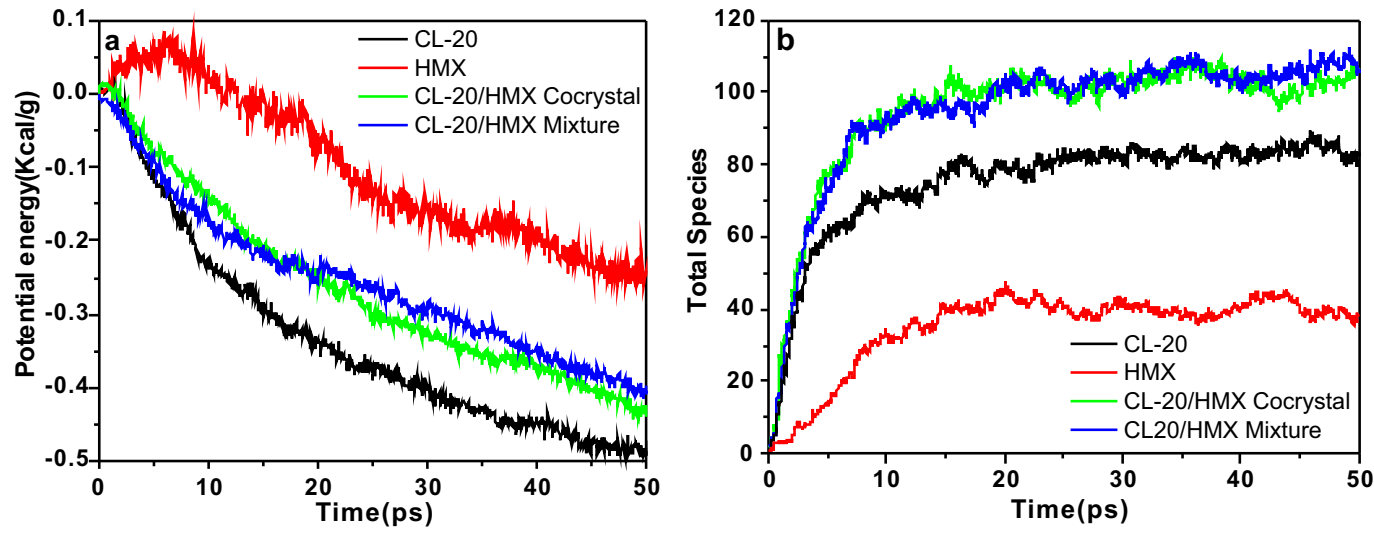

Fig. 3 The potential energy (a) and total species (b) changes along with the time evolution. (The initial potential energy is set to be zero as a reference). 
study the thermal decomposition mechanism in 2000 K. Fig. 3a exhibits the evolution of the potential energy of different systems with the change of simulation time. Clearly, the potential energy of HMX system shows no significant descending tendency with the comparison of the others at initial simulation time (10 ps). For CL20 and its mixture and cocrystal, with the increase of time, the total potential energy decreased sharply in the whole of simulation times because of the higher thermal sensitivity and thermal decomposition of CL-20. The potential energy curve of pure CL-20 crystal system shows a steeper tendency than those of the CL-20/HMX mixture and cocrystal. The reason is that some of CL- 20 molecules in the mixture and cocrystal are isolated by relatively stable HMX molecules, which make the sensitive CL-20 molecules less likely to interact with other CL-20 molecules for the following chemical reactions and restrict the rapid decomposition. In contrast to CL-20/HMX mixture, CL-20/HMX cocrystal need a fusion process of cocrystal at the initial stage of the induction (endothermic) decomposition due to endothermic $\mathrm{NO}_{2}$ dissociation, which result in the higher potential energy. After 20 ps, the CL-20/HMX cocrystal decomposed rapidly, and released plenty of energy.

Fig. $3 \mathrm{~b}$ shows the total number of species in the reaction process along with the simulate time evolution. HMX has a lower thermal sensitivity, thus there is not any thermal decomposition reaction within the first $10 \mathrm{ps}$, resulting in the few number of species. While, CL-20 crystal has generated a large number of products within 5 ps, then fluctuated around 20 ps. The pure CL-20 and HMX system have less atom number (1152 atoms for CL-20and 512 atoms for HMX) in the initial state, so the mixture and CL-20/HMX cocrystal produce more species than CL-20 and HMX system. Strong intermolecular interactions will promote the thermal decomposition reaction of the components in the system, whereas the specific mechanical chemical coupling mechanism needs to be further studied.

\subsection{Species analysis}

Fig. 4 demonstrates the detailed species analysis of the CL-20/HMX cocrystal, CL-20/HMX mixture, CL-20 and HMX at the temperature of $2000 \mathrm{~K}$ along with the time evolution. The formation of several key products and intermediates of CL-20 and HMX decomposition are observed, such as $\mathrm{NO}_{2}, \mathrm{~N}_{2}, \mathrm{NO}, \mathrm{H}_{2} \mathrm{O}, \mathrm{HONO}, \mathrm{HON}$ and $\mathrm{CO}_{2}$, which are in accordance with the experimental results. ${ }^{36}$

The primary decomposition products of $\mathrm{NO}_{2}$, were formed faster in larger quantities. $\mathrm{NO}_{2}$ was derived from the dissociation of $\mathrm{N}-\mathrm{NO}_{2}$ bond owing to the thermal decomposition of CL-20, in agreement with the characteristic initiation thermolysis reaction of cyclic nitramines. ${ }^{32} \mathrm{~N}_{-} \mathrm{NO}_{2}$ is also the initiation bond of $\mathrm{HMX}$ thermolysis, which is a route to produce $\mathrm{NO}_{2}$. Subsequently, much $\mathrm{NO}_{2}$ would be consumed away by the secondary reaction, so the number of $\mathrm{NO}_{2}$ decreased rapidly and $\mathrm{NO}, \mathrm{HONO}, \mathrm{HNO}_{3}$ etc. would be generated.

$\mathrm{NO}$ is also the main product of thermolysis, and the formation paths are shown as follows. $\mathrm{N}_{2} \mathrm{O}_{4}$ was created by two $\mathrm{NO}_{2}$ molecular fragments, then it was decomposed into $\mathrm{NO}_{3}$ and $\mathrm{NO}$. N-N bond in CL-20 and HMX was dissociated to generate C-ONO group, which arranged and acquired a proton to form HONO, leading to the generation of NO. It agrees well with the results of Isayev. ${ }^{37}$ With similar to $\mathrm{NO}_{2}$, $\mathrm{NO}$ fission shaped in the early stages of thermal decomposition, then generated $\mathrm{N}_{2}$ due to the secondary reaction.

The generation paths of $\mathrm{H}_{2} \mathrm{O}$ are described as follows: at initial stage, $\mathrm{NOOH}$ forms through nitro-group; then, it captures a proton to form $-\mathrm{NOOH}-\mathrm{H}$; and $1 \mathrm{~mol}$ of $\mathrm{H}_{2} \mathrm{O}$ is pulled off. Second, $\mathrm{NO}_{2}$ is
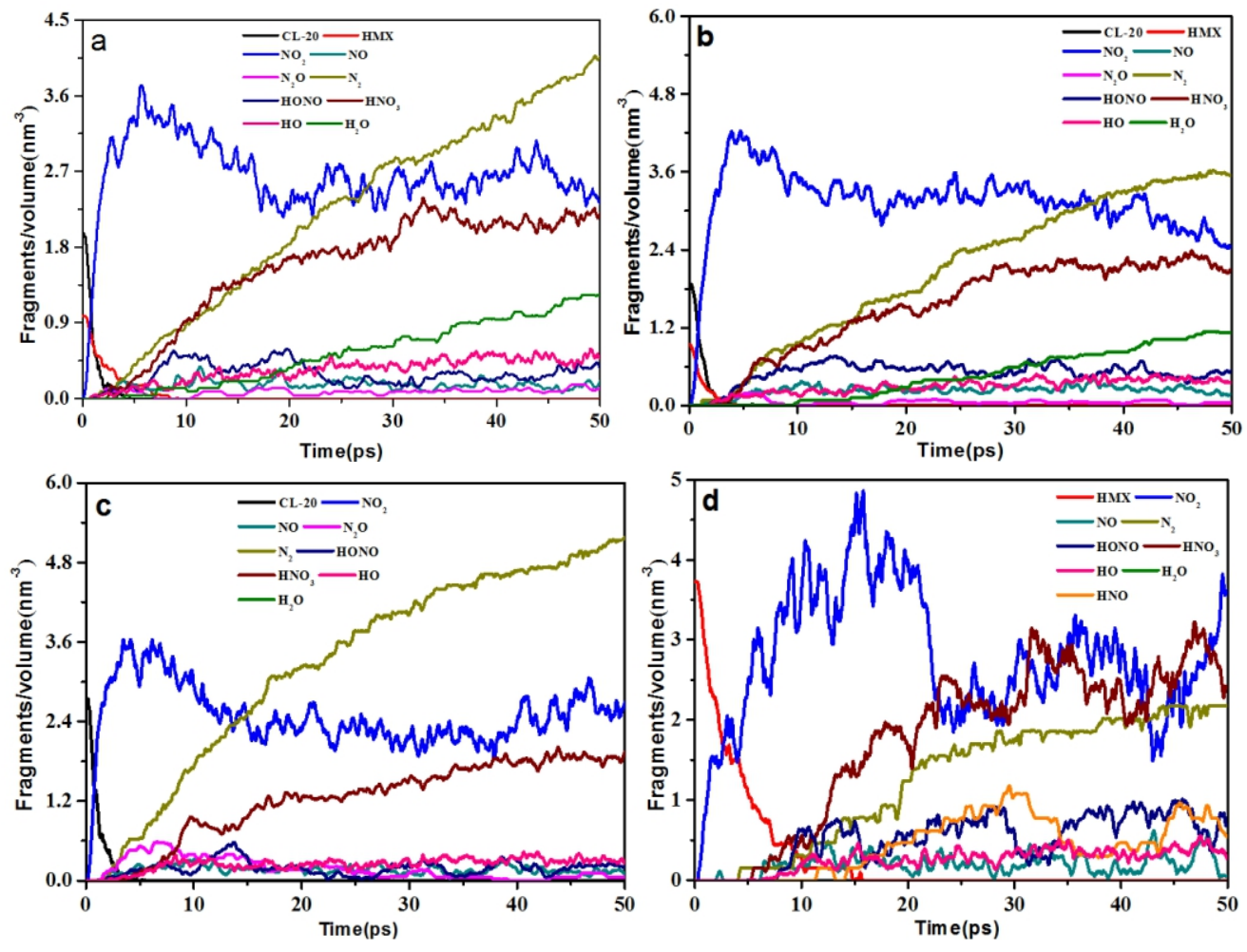

Fig. 4 The distribution of main products in (a) cocrystal, (b) mixture of CL-20 and HMX, (c) pure CL-20 and (d) HMX along with the time evolution. 
arranged in forms of ONO-. Then it seizes a proton to form HONO. HONO is thermally decomposed to HO- and promotes the generation of $\mathrm{H}_{2} \mathrm{O}$. Third, $\mathrm{HNO}_{3}$ is thermally decomposed to $\mathrm{H}_{2} \mathrm{O}$.

Few HONO is produced at the early stage in the CL-20 decomposition, which are successively dominated by $\mathrm{NO}_{2}$ fission and ring-breaking. No HONO is formed during the later stages of the reaction, probably due to the abstraction of a hydrogen atom of $\mathrm{NO}_{2}$ from other free radical and intermediates. In the condition, the HONO molecule is not stable and rapidly dissociated into NO and $\mathrm{OH}$ radicals.

Although the underpinning mechanism of decomposition reaction remains unclear, based on the analysis of the decomposition products and previously reported work, ${ }^{37,38}$ we can infer that the possible decomposition reactions may undergo three-steps, (1) unimolecular endothermic decomposition of the CL-20 or HMX molecular by breaking $\mathrm{N}-\mathrm{NO}_{2}$ trigger bond in CL-20 and $\mathrm{N}-\mathrm{NO}_{2}$ trigger bond in HMX molecules. (2) unimolecular decomposition of primary fragments into such intermediates as $\mathrm{N}_{2} \mathrm{O}, \mathrm{HONO}, \mathrm{HNO}_{2}$, etc. (3) gas-phase reaction to produce final stable products $\left(\mathrm{N}_{2}, \mathrm{NO}\right.$, $\mathrm{H}_{2} \mathrm{O}$, etc.).

\section{Conclusions}

Molecular dynamics simulation method was applied to study the structure and property of pure CL-20, HMX, CL-20/HMX mixture and cocrystal. The sensitivity, mechanical property and thermal decomposition process were analyzed. The mixture and CL-20/HMX cocrystal made the maximum bond length of trigger bond $\mathrm{N}-\mathrm{NO}_{2}$ in CL-20 become shorter and $\mathrm{N}-\mathrm{NO}_{2}$ in $\mathrm{HMX}$ elongated. The mixture and CL-20/HMX cocrystal decreased the sensitivity of CL-20. The binding between CL-20 and HMX of CL-20/HMX cocrystal was far stronger than that of CL-20/HMX mixture, and the CL-20/HMX cocrystal structure was more stable than their mixture. Cocrystal and mixture decreased the stiffness and made CL-20 system more flexible and "softer". The cocrystal and mixture reduce the "hot spot" formation and sensitivity of CL-20 explosive, increasing the security of CL-20. The several key products and intermediates of CL20/HMX mixture and cocrystal decomposition are $\mathrm{NO}_{2}, \mathrm{~N}_{2}, \mathrm{NO}$, $\mathrm{H}_{2} \mathrm{O}, \mathrm{HONO}, \mathrm{HON}$ and $\mathrm{CO}_{2}$.

\section{Conflict of interest}

There are no conflicts to declare.

\section{Acknowledgements}

Project is supported by the Specialized Research Fund for the Doctoral Program of Higher Education of China (Grant No.20131420120004). Science Foundation for Young Scientists of Shanxi Province(Grant No.201701D221103).

\section{References}

1. A. T. Nielsen, A. P. Chafin, S. L. Christian, D. W. Moore, M. P. Nadler, R. A. Nissan, D. J. Vanderah, R. D. Gilardi, C. F. George and J. L. Flippen-Anderson, Tetrahedron, 1998, 54, 11793-11812.

2. Y. A. Bogdanova, S. A. Gubin, B. L. Korsunskii and V. I. Pepekin, Combust. Explo. Shock, 2009, 45, 738-743.

3. P. A. U. R. L. Simpson, D. L. Ornellas, G. L. Moody, K. J. Scribner, and D. M. Hoffman, Propellants Explos. Pyrotech., 1997, 249-255.

4. J. P. Agrawalt, Energy Cornbust., 1998, 24, 1-30.
5. A. K. Sikder and N. Sikder, J. Hazard. Mater, 2004, 112, 1-15.

6. J.-M. Lehn, Angew. Chem. Int. Ed. Engl., 1988, 27, 89-112.

7. F. Lara-Ochoa and G. Espinosa-Petrez, Supramol. Chem., 2007, 19, 553-557.

8. Y. P. Wang, Z. W. Yang, H. Z. Li, X. Q. Zhou, Q. Zhang, J. H. Wang and Y. C. Liu, Propellants Explos. Pyrotech., 2014, 39, 590-596.

9. O. Bolton and A. J. Matzger, Angew. Chem. Int. Ed. Engl., 2011, 50, 8960-8963.

10. Z. W. Yang, H. Z. Li, X. Q. Zhou, C. Y. Zhang, H. Huang, J. S. Li and F. D. Nie, Cryst. Growth Des., 2012, 12, 5155-5158.

11. H. F. Xu, X. H. Duan, H. Z. Li and C. H. Pei, Rsc Adv., 2015, 5, 95764-95770.

12. C. Y. Guo, H. B. Zhang, X. C. Wang, J. J. Xu, Y. Liu, X. F. Liu, H. Huang and J. Sun, J. Mol. Struct., 2013, 1048, 267-273.

13. O. Bolton, L. R. Simke, P. F. Pagoria and A. J. Matzger, Cryst. Growth Des., 2012, 12, 4311-4314.

14. N. Rom, S. V. Zybin, A. C. van Duin, W. A. Goddard, 3rd, Y. Zeiri, G. Katz and R. Kosloff, J. Phys. Chem. A, 2011, 115, 10181-10202.

15. S. P. Han, A. C. van Duin, W. A. Goddard, 3rd and A. Strachan, J. Phys. Chem. B, 2011, 115, 6534-6540.

16. A. Strachan, E. M. Kober, A. C. van Duin, J. Oxgaard and W. A. Goddard, J. Chem. Phys., 2005, 122, 54502.

17. M. R. Weismiller, M. F. Russo, A. C. T. van Duin and R. A. Yetter, Proc. Combust. Inst., 2013, 34, 3489-3497.

18. A. Strachan, A. C. van Duin, D. Chakraborty, S. Dasgupta and W. A. Goddard, 3rd, Phys. Rev. Lett., 2003, 91, 098301.

19. Z. Li, C. Lang, W. Chen and W. Jun-ying, Chin. J. of Explos. Propellants, 2012, 4, 002.

20. H. Liu, Q. K. Li and Y. H. He, Acta Phys Sin.-Ch Ed, 2013, 62.

21. D. Z. Guo, Q. An, S. V. Zybin, W. A. Goddard, F. L. Huang and B. Tang, J. Mater. Chem. A, 2015, 3, 5409-5419.

22. H. Sun, J. Phys. Chem. B, 1998, 102, 7338-7364.

23. L. Liu, Y. Liu, S. V. Zybin, H. Sun and W. A. Goddard, 3rd, J. Phys. Chem. A, 2011, 115, 11016-11022.

24. D. I. A. Millar, H. E. Maynard-Casely, A. K. Kleppe, W. G. Marshall, C. R. Pulham and A. S. Cumming, CrystEngComm, 2010, 12, 2524-2527.

25. H. H. Cady, A. C. Larson and D. T. Cromer, Acta Crystallogr., 1963, 16, 617-623.

26. H. C. Andersen, J. Chem. Phys., 1980, 72, 2384-2393.

27. H. J. C. Berendsen, J. P. M. Postma, W. F. van Gunsteren, A. DiNola and J. R. Haak, J. Chem. Phys., 1984, 81, 3684-3690.

28. P. P. Ewald, Ann. Phys., 1921, 369, 253-287.

29. M. P. Tosi, Solid State Phys., 1964, 16, 1-120.

30. W. Zhu, J. J. Xiao, J. Zheng, X. B. Zhao, Z. E. Chen and H. M. Xiao, Acta Chim. Sinica, 2008, 66, 2592-2596.

31. T. Su, R. Peng, Z. Hood, M. Naguib, I. Ivanov, J. Keum, Z. Qin, Z. Guo and Z. Wu, ChemSusChem, 2018, 11, 688-699.

32. X. XiaoJuan, X. Jijun, H. Hui, L. JinShan and X. HeMing, Sci. China Ser. B-Chem., 2007, 50, 737-745.

33. Z.-z. Qin, T.-m. Su, H.-b. Ji, Y.-x. Jiang, R.-w. Liu and J.-h. Chen, Aiche J., 2015, 61, 1613-1627.

34. T. Su, Q. Shao, Z. Qin, Z. Guo and Z. Wu, ACS Catal., 2018, 8, 2253-2276.

35. T. Sun, Q. Liu, J. J. Xiao, F. Zhao and H. M. Xiao, Acta Chim. Sinica, 2014, 72, 1036-1042.

36. J. C. Oxley, A. Kooh, R. Szekeres and W. Zheng, J. Phys. Chem., 
1994, 98, 7004-7008.

37. O. Isayev, L. Gorb, M. Qasim and J. Leszczynski, J Phys. Chem. $B, 2008,112,11005-11013$.
38. L. Z. Zhang, S. V. Zybin, A. C. T. van Duin, S. Dasgupta, W. A. Goddard and E. M. Kober, J. Phys. Chem. A, 2009, 113, 1061910640. 